Check for updates

Cite this: Chem. Sci., 2019, 10, 6815

๑ All publication charges for this article have been paid for by the Royal Society of Chemistry

\section{Dual resonance energy transfer in triple- component polymer dots to enhance electrochemiluminescence for highly sensitive bioanalysis $\uparrow$}

\author{
Ningning Wang, ${ }^{a}$ Ziyu Wang, ${ }^{b}$ Lizhen Chen, ${ }^{a}$ Weiwei Chen, ${ }^{a}$ Yiwu Quan, \\ Yixiang Cheng (D) *b and Huangxian Ju (DD *a
}

Polymer dots (Pdots) have become a type of attractive illuminant for electrochemiluminescence (ECL). However, the low ECL efficiency severely limits their practicability. Here, we design a dual intramolecular resonance energy transfer (RET) mechanism with newly synthesized triple-component Pdots to achieve great ECL enhancement. This mechanism efficiently shortens the path of energy transmission, thus greatly promoting the ECL amplification by 380 and 31 times compared to systems with no and single $\mathrm{RET}$, and results in a relative $\mathrm{ECL}$ efficiency of $23.1 \%$ (vs. $1 \mathrm{mM} \mathrm{Ru(bpy)} 3^{2+}$ ). Using metal-organic frameworks to carry the triple-component Pdots, a highly luminescent probe is proposed. By integrating the probe with target-mediated enzymatic circulation amplification and DNA arrays, a highly sensitive ECL imaging method is designed for simultaneous visual analysis of two kinds of proteins, mucin 1 and human epidermal growth factor receptor 2 , on living cells, which exhibited linear ranges of $1 \mathrm{pg} \mathrm{mL}^{-1}$ to $5 \mathrm{ng} \mathrm{mL}-1$ and $5 \mathrm{pg} \mathrm{mL}^{-1}$ to $10 \mathrm{ng} \mathrm{mL}-1$ with limits of detection of $1 \mathrm{pg} \mathrm{mL}^{-1}$ and $5 \mathrm{pg} \mathrm{mL}^{-1}$, respectively. The proposed strategy showed promising application in bioanalysis.
Received 31st March 2019

Accepted 28th May 2019

DOI: $10.1039 / c 9 s c 01570 a$

rsc.li/chemical-science particular, the AIE-active ECL luminophores have been regarded as one of the most interesting members due to their amplification effect in aggregate states. ${ }^{17}$ Moreover, intramolecular D-A transfer can activate tunable ECL signals, which provides an alternative pathway to improve the ECL response. Inspired by these results, in our previous work we synthesized two kinds of three-component Pdots with the same AIE-active moiety but different D-A structures containing carbazole and fluorene, respectively, and proved the stronger electrondonating ability of the carbazole moiety with a lower ECL onset potential and higher intensity of ECL emission. ${ }^{18}$ To achieve excellent ECL performance, this work further screened optimal types of the acceptor and an appropriate ratio of D/A for the preparation of Pdots, and designed a dual resonance energy transfer (RET) (D1-A1/D2-A2) mechanism to overcome the bottleneck of Pdots in ECL efficiency.

As well known, ECL-RET from the donor to acceptor, similar to Förster RET (FRET), has demonstrated its ability to extend ECL applications to biosensing ${ }^{\mathbf{1 9 , 2 0}}$ and monitoring molecular interactions ${ }^{21}$ owing to potential and spatial control. ${ }^{22,23}$ By incorporating $\mathrm{Ru}(\mathrm{bpy})_{3}{ }^{2+}$ into Pdots, the RET from the excited Pdots to encapsulated $\mathrm{Ru}(\mathrm{bpy})_{3}{ }^{2+}$ can enhance the ECL emission by 15.7 fold. ${ }^{24}$ The intramolecular RET achieved by integrating luminol (donor) and $\mathrm{Ru}(\mathrm{bpy})_{2}(\mathrm{mcpbpy})^{2+}$ (acceptor) into one molecule has showed enhanced ECL emission owing to the short path of energy transmission and less energy loss. ${ }^{25} \mathrm{In}$ this
${ }^{a}$ State Key Laboratory of Analytical Chemistry for Life Science, School of Chemistry and Chemical Engineering, Nanjing University, Nanjing 210023, China. E-mail: hxju@nju. edu.cn

${ }^{b}$ Key Lab of Mesoscopic Chemistry of MOE, Jiangsu Key Laboratory of Advanced Organic Materials, School of Chemistry and Chemical Engineering, Nanjing University, Nanjing 210023, China.E-mail: yxcheng@nju.edu.cn

$\dagger$ Electronic supplementary information (ESI) available: Experimental section, supporting figures and tables. See DOI: 10.1039/c9sc01570a 
A
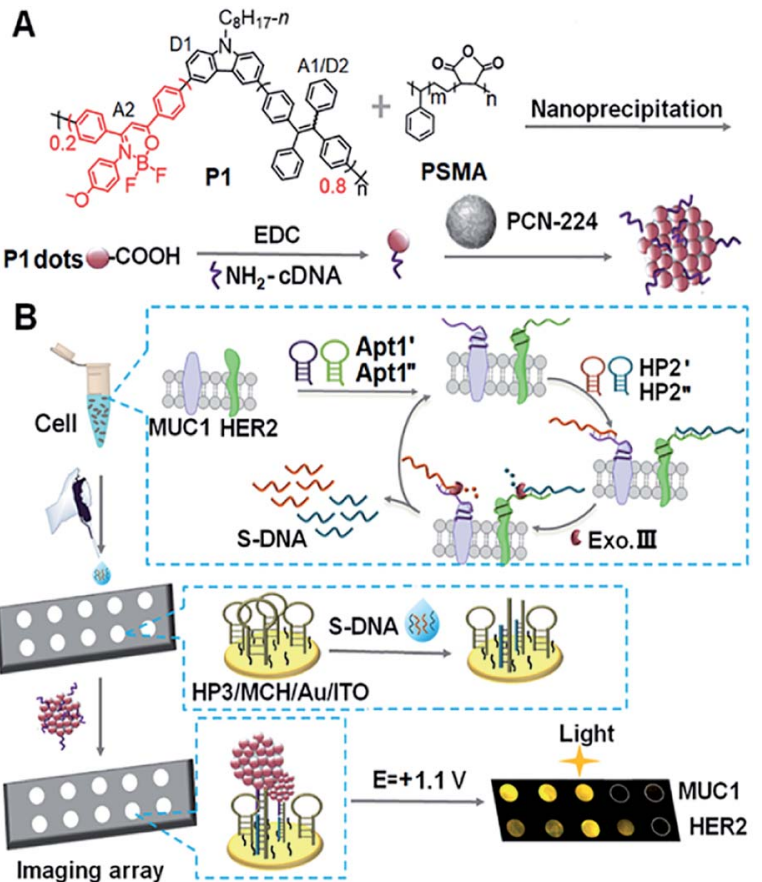

Scheme 1 Schematic diagram of (A) preparation of Pdots@PCN-224 as an ECL probe and (B) ECL imaging array for the analysis of two kinds of membrane proteins with target-mediated cyclic amplification.

work, triple-component (D1, A1/D2 and A2) Pdots (P1 dots) are composited with carbazole, tetraphenylethene (TPE), and 2,2difluoro-3-(4-methoxyphenyl)-4,6-diphenyl-2H-1,3l4,2l4-

oxazaborinine (DMDO) (Scheme 1A). The carbazole moiety is electroactive and can be oxidized at a relatively low potential to produce the excited state and act as D1. The energy is then transferred to the TPE moiety that acts as both A1 and D2 to transfer the energy to DMDO (A2). The short path of energy transmission greatly promotes ECL amplification for the preparation of the sensitive ECL probe. By integrating the probe with target-mediated enzymatic circulation amplification and DNA arrays (Scheme 1B), the P1 dots have successfully been used for highly sensitive ECL imaging. Therefore, a simultaneous visual detection method for two kinds of membrane proteins, mucin 1 (MUC1) and human epidermal growth factor receptor 2 (HER2), on living cells was developed with specific recognition and DNA coding technology. The excellent performance of the proposed method indicates the promising application of the dual RET mechanism along with the triple-component Pdots in bioanalysis.

\section{Results and discussion}

\section{Design and characterization of P1-P4 and Pdots}

In the dual intramolecular RET mechanism, AIE-active TPE plays key roles (A1/D2). As the proof-of-concept, three model polymers (P2-P4) were designed (Fig. 1A). Specifically, P2 included the moieties of D1 and A1/D2 with single ECL-RET, while P3 consisted of A2 without ECL-RET. Besides, P4 was composed of more A1/D2 and less A2 compared to P1 and acted as the model of
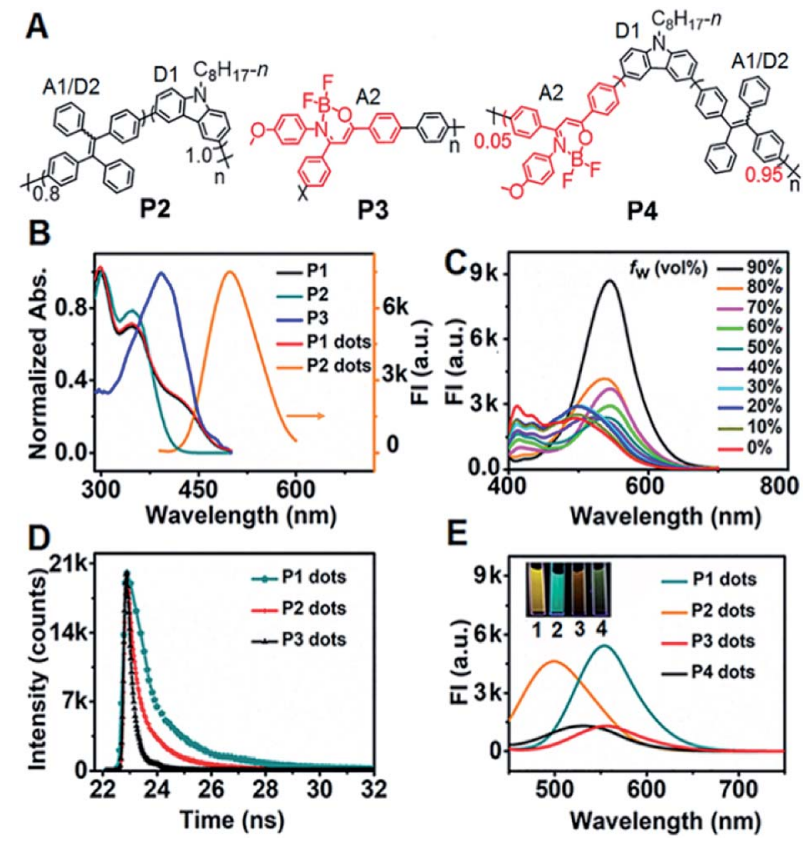

Fig. 1 (A) Structural formulae of P2-P4. (B) UV-vis absorbance of P1$\mathrm{P} 3$ in THF and $\mathrm{P} 1$ dots in $\mathrm{H}_{2} \mathrm{O}$, and the FL spectrum of $\mathrm{P} 2$ dots in $\mathrm{H}_{2} \mathrm{O}$ at a $\lambda_{\text {ex }}$ of $370 \mathrm{~nm}$. (C) FL spectra of $\mathrm{P} 1$ in $\mathrm{THF}-\mathrm{H}_{2} \mathrm{O}$ mixtures with different contents of $\mathrm{H}_{2} \mathrm{O}$ at a $\lambda_{\text {ex }}$ of $370 \mathrm{~nm}$. (D) FL lifetimes of P1-P3 dots in $\mathrm{H}_{2} \mathrm{O}$ at a $\lambda_{\text {ex }}$ of $374 \mathrm{~nm}$. (E) FL spectra of $10 \mu \mathrm{g} \mathrm{mL}^{-1} \mathrm{P} 1$ dots, 50 $\mu \mathrm{g} \mathrm{mL}^{-1} \mathrm{P} 2$ dots, $100 \mu \mathrm{g} \mathrm{mL}^{-1} \mathrm{P} 3$ dots, and $50 \mu \mathrm{g} \mathrm{mL}^{-1} \mathrm{P} 4$ dots at a $\lambda_{\text {ex }}$ of $370 \mathrm{~nm}$. Inset: FL photos of P1-P4 dots.

incomplete dual ECL-RET. In P1, the optimal ratio of A1/D2 to A2 was $8: 2$ (Fig. S1†). The detailed synthetic routes of P1-P4 were modified according to our previous reports (Scheme $\mathrm{S} 1 \dagger$ ). ${ }^{26-28}$ The Pdots could be prepared by a nanoprecipitation method utilizing a polymer as the luminescent precursor and poly(styrene-comaleic anhydride) (PSMA) as a functional reagent.

P1 exhibited three distinct absorption peaks in THF solution centered at 300, 349 and $429 \mathrm{~nm}$, respectively (Fig. 1B). The peaks at 300 and $349 \mathrm{~nm}$ also occurred in the absorption spectrum of P2. Compared with $\mathbf{P 2}$ and P3, the shoulder absorption peak at $429 \mathrm{~nm}$ could be assigned to the DMDO chromophore (A2), indicating the intramolecular charge transfer process from the electron-rich carbazole donor to the electron-deficient DMDO acceptor via the TPE bridge linker. ${ }^{29}$ The identifiable overlap of the absorption of P1 dots with the emission of $\mathbf{P 2}$ dots in $\mathrm{H}_{2} \mathrm{O}$ demonstrated the feasibility of the second FRET process from the TPE moiety (A1/D2) to DMDO (A2) (Fig. 1B).

The fluorescence spectrum of P1 in THF showed a weak emission situated at $412 \mathrm{~nm}$ with a shoulder peak at $433 \mathrm{~nm}$ (Fig. 1C), which could be attributed to the carbazole moiety. Upon addition of $\mathrm{H}_{2} \mathrm{O}$ to THF, these peaks gradually decreased and disappeared until the water fraction $\left(f_{\mathrm{w}}\right)$ reached $70 \%$. Meanwhile, the peak at $496 \mathrm{~nm}$ due to the emission of the TPE moiety gradually increased from $f_{\mathrm{w}}=0$ to $30 \%$, and then disappeared at $f_{\mathrm{w}}=40 \%$, indicating the first intramolecular FRET process from the carbazole moiety to the TPE moiety. ${ }^{18}$ With increasing $f_{\mathrm{w}}$, a new emission peak assigned to the DMDO 
chromophore appeared at $527 \mathrm{~nm}$, which gradually redshifted to $548 \mathrm{~nm}$ at a $f_{\mathrm{w}}$ of $90 \%$ with an abrupt increase of the peak intensity at $f_{\mathrm{w}}$ in the range of $80 \%$ to $90 \%$, demonstrating the presence of the second intramolecular FRET process in P1 dots at high water fractions.

The P1 dots showed a fluorescence lifetime $\tau$ of $8.00 \mathrm{~ns}$ (CHISQ $=1.03$ ), which was much longer than $1.58 \mathrm{~ns}$ of $\mathbf{P 2}$ dots $(\mathrm{CHISQ}=1.00)$ (Fig. 1D), demonstrating the different fluorescent emission moieties and the presence of the second FRET in P1 dots. The fluorescence lifetime of $2.68 \mathrm{~ns}(\mathrm{CHISQ}=1.09)$ for P3 dots was also slightly longer than that of $\mathbf{P 2}$ dots. Therefore, the fluorescence spectra of $\mathbf{P 1}$ and $\mathbf{P 3}$ dots exhibited different emission peak wavelengths from that of $\mathbf{P} 2$ dots (Fig. 1E). When the fraction of DMDO decreased to 0.05 , a weak emission peak appeared at $530 \mathrm{~nm}$, indicating the weakened FRET in P4 dots due to an incomplete dual RET, which also demonstrated the FRET effect from TPE to DMDO.

\section{Dual ECL-RET of P1 dots}

In the presence of tri- $n$-propylamine (TPrA) as a co-reactant, a P1 dot modified glassy carbon electrode (GCE) showed the maximum ECL intensity, compared with the responses in the presence of other coreactants such as $\mathrm{K}_{2} \mathrm{C}_{2} \mathrm{O}_{4}, \mathrm{Na}_{2} \mathrm{SO}_{3}$ and TEA (Fig. S2 $\dagger$ ). The ECL intensity increased with increasing concentration of TPrA and then decreased to a steady value at $25 \mathrm{mM}$ (Fig. S3 $\dagger$ ). Thus $25 \mathrm{mM}$ TPrA was chosen as the optimal amount of the anodic co-reactant. TPrA showed a broad irreversible oxidation peak at $+1.02 \mathrm{~V}$ with an onset potential of $+0.57 \mathrm{~V}$ at the GCE (Fig. 2A). ${ }^{30}$ Meanwhile, P1 dots|GCE in PBS showed an oxidation current, indicating that $\mathbf{P 1}$ dots were electrochemically oxidized to form cationic radicals by injecting holes into the highest occupied molecular orbital. ${ }^{31}$ As a result, the ECL emission of P1 dots $\mid$ GCE occurred at $+1.12 \mathrm{~V}$ with the onset potential of $+0.89 \mathrm{~V}$ in the presence of TPrA (Fig. 2B). The ECL spectra of P1-P4 dots coincided well with their fluorescence spectra (Fig. 2C), indicating the presence of the same excited species in ECL emission as those in fluorescence emission. ${ }^{32}$ Similar to the FRET enhancement in fluorescence emission, the enhanced ECL emission of P1 dots|GCE compared with P2-P4 dots|GCE (Fig. 2D) was caused by the RET process. Moreover, the onset potential of $+0.89 \mathrm{~V}$ for P1 dots $\mid$ GCE was similar to that of $\mathbf{P 2}$ dots $(+0.87 \mathrm{~V})$ and $\mathbf{P 4}$ dots $(+0.93 \mathrm{~V})$ but much lower than that of $\mathbf{P 3}$ dots $(+1.13 \mathrm{~V})($ Fig. 2D). These phenomena were further evidence of the detailed mechanism of dual RET in P1 dots (Fig. 2E): TPrA was first oxidized and deprotonated to produce a reducing agent (TPrA $\left.{ }^{\circ}\right)$ at potentials more positive than $+0.57 \mathrm{~V}$. Afterward, the electrons were injected into oxidized Pdots ${ }^{\cdot+}\left(\mathrm{D} 1{ }^{\cdot+}-\mathrm{A} 1 / \mathrm{D} 2-\mathrm{A} 2\right)$, which produced the excited Pdots* (D1*-A1/D2-A2), and the energy was transferred to TPE and then to DMDO to generate excited D1-A1/D2-A2* for ECL emission. As a result, the ECL intensity for P1 was about 31- and 380-fold higher than those of single (P2) and no RET (P3) systems, respectively (Fig. 2D).

The relative ECL efficiency of $50 \mu \mathrm{g} \mathrm{mL} \mathrm{m}^{-1} \mathbf{P 1}$ dots $v s .1 \mathrm{mM}$ $\mathrm{Ru}(\mathrm{bpy})_{3}{ }^{2+}$ was calculated to be $23.1 \%$ (Fig. S4†), which was obviously higher than those of commercial poly[2-methoxy-5-(2-
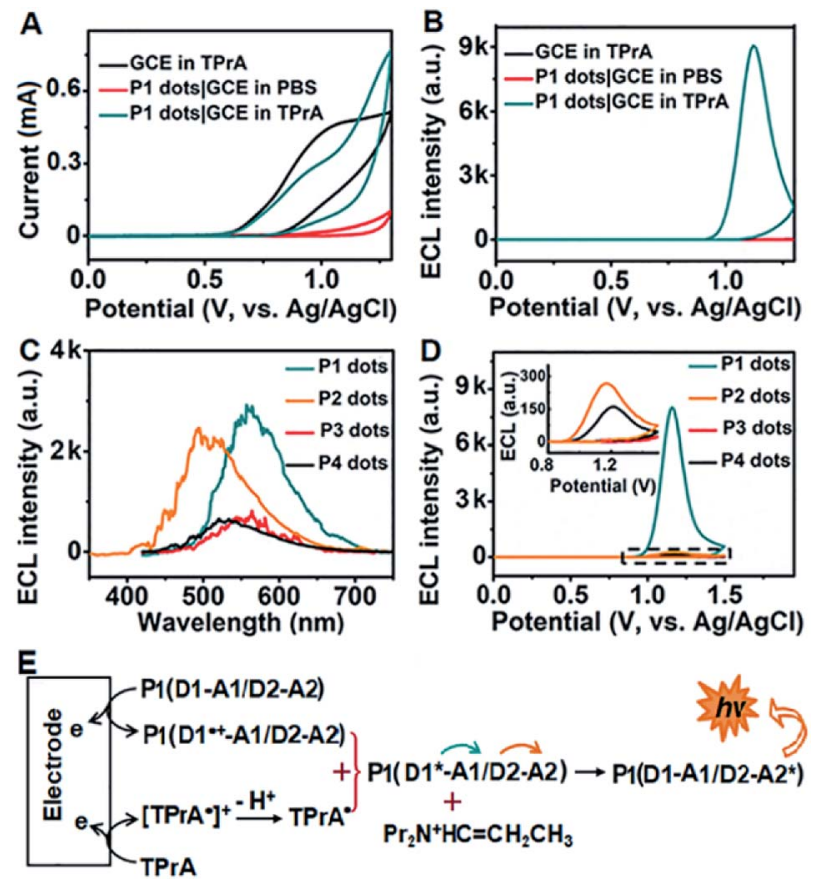

Fig. 2 (A) CV and (B) ECL curves of bare and P1 dot modified GCE in $0.1 \mathrm{M} \mathrm{pH}$ 7.4 PBS in the presence and absence of $25 \mathrm{mM}$ TPrA as the co-reactant. (C) ECL spectra of $10 \mu \mathrm{g} \mathrm{mL}^{-1} \mathrm{P} 1$ dots, $50 \mu \mathrm{g} \mathrm{mL}^{-1} \mathrm{P} 2$ dots, $100 \mu \mathrm{g} \mathrm{mL}^{-1} \mathrm{P} 3$ dots, and $50 \mu \mathrm{g} \mathrm{mL}^{-1} \mathrm{P} 4$ dots in $100 \mathrm{mM}$ TPrA. (D) ECL curves of $50 \mu \mathrm{g} \mathrm{mL}^{-1} \mathrm{P} 1-\mathrm{P} 4$ dots|GCE in $25 \mathrm{mM}$ TPrA. Inset: enlarged view of the dotted line region. PMT: $300 \mathrm{~V}$. Scan rate: $100 \mathrm{mV}$ $\mathrm{s}^{-1}$. (E) ECL mechanism of P1 dots in TPrA (P1 represents P1 dots).

ethyl hexyloxy)-1,4-(1-cyanovinylene-1,4-phenylene)] Pdots $(11.22 \%)^{12}$ and previously reported triple-component Pdots (Table S1†). The ECL emission of P1 dots also showed the lowest onset and peak potentials, compared with several commercial Pdots (Fig. S5 and Table S2 $\uparrow$ ), demonstrating the distinguished performance of $\mathbf{P 1}$ dots in ECL emission.

To assess the intrinsic energy parameters and infer the relative stability of electron/hole injected $\mathbf{P 1}$ dots, annihilation ECL was performed in the absence of TprA. As expected, P1 dots could be electrochemically oxidized to form cation radicals or reduced to anion radicals in PBS by injecting holes or electrons into P1 dots (Fig. S6A $\dagger$ ), ${ }^{33}$ which led to a strong annihilation ECL emission with a peak at $+1.07 \mathrm{~V}$ during anodic scanning, and a weak ECL signal in the cathodic region with an onset potential of $-1.30 \mathrm{~V}$, respectively (Fig. S6B $\dagger$ ). No matter in the oxidative or reductive process-initiated ECL transients, distinguished ECL only appeared at $+1.10 \mathrm{~V}$ (Fig. S6C and $\mathrm{D} \uparrow$ ), demonstrating that the radical for the anodic ECL emission was more stable. ${ }^{34}$ In addition, considering that the same excited species are present in both annihilation and co-reactant ECL emission, the ECL band gap of P1 dots could be estimated from the onset potentials of anodic and cathodic annihilation ECL emission at +0.84 and $-1.30 \mathrm{~V}$ to be $2.14 \mathrm{eV}$ (Fig. S6B $\dagger$ ). This was consistent with the energy level $(2.16 \mathrm{eV})$ from CV of P1 that gave quasireversible oxidation peaks with an onset potential of $+0.78 \mathrm{~V}$ resulting from the oxidation of the TPE moiety ${ }^{35}$ and an onset reduction potential of $-1.38 \mathrm{~V}$ (Fig. S7†). 


\section{Target-mediated enzymatic circulation amplification}

The designed P1 dots with a high ECL efficiency showed very low cytotoxicity (Fig. S8†), providing promising application in bioanalysis. Using two kinds of tumor-associated membrane proteins, MUC1 and HER2, as model targets, Apt1 for coding the targets by the specific recognition of aptamers to proteins, HP2 for performing target-mediated enzymatic circulation amplification, HP3 for capturing the released codes (S-DNA) on a DNA array, and capture DNA (cDNA) for labelling the ECL probe (P1 dots@PCN-224), a sensitive ECL imaging platform was developed. The detailed DNA sequences are listed in Table S3. $\dagger$ As shown in Fig. $3 \mathrm{~A}$ and $\mathrm{B}$, the recognition of aptamers to proteins induced the hybridization of Apt1 with HP2 (lines a and b), which produced a band at low migration, and exonuclease III (Exo III) could digest the Apt1/ HP2 duplex to produce the S-DNA with a band at high migration (line c), which hybridized with HP3 to form a new band (line $\mathrm{d}$ ). The hybridization product of S-DNA and HP3 could be recognized by cDNA to form another band at relatively low migration (line e). These phenomena verified the feasibility of the designed imaging strategy, including coding, amplification, and capture processes. Moreover, the hybridization between HP3 and cDNA, as well as among Apt1, HP2 and HP3, in the absence of the target could be excluded (lines $f$ and $g$ ).

The specific recognition of Apt1 toward targets was further demonstrated with confocal laser scanning microscopy (CLSM) imaging on the living cell surface (Fig. 3C and D). Compared to the treatment with random 1-FAM and random 2-FAM, bright fluorescence was observed on both MUC1-over-expressed MCF7 cells $^{36}$ upon incubation with Apt1'-FAM and HER2-overexpressed SK-BR-3 cells ${ }^{37}$ upon incubation with Apt1"-FAM, respectively.

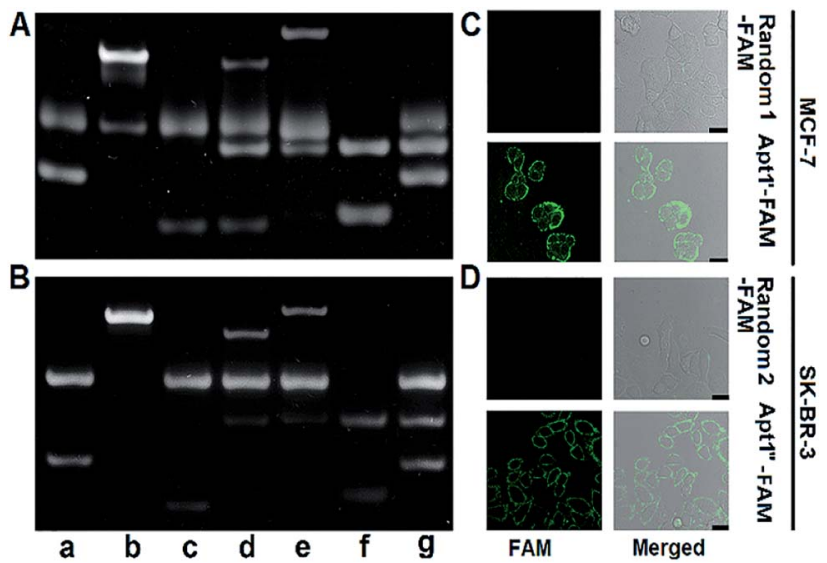

Fig. 3 PAGE images for verifying the feasibility of (A) MUC1 and (B) HER2-mediated enzymatic circulation amplification. (a) Apt1 + HP2, (b) (a) + MUC1 or HER2, (c) (b) + Exo III, (d) (c) deactivated at $85^{\circ} \mathrm{C}$ for $10 \mathrm{~min}+\mathrm{HP3}$, (e) (d) + CDNA, (f) HP3 + cDNA, and (g) Apt1 + HP2 + HP3. CLSM images of (C) MCF-7 cells after incubation with $1 \mu \mathrm{M}$ random 1-FAM and Apt1'-FAM, and (D) SK-BR-3 cells after incubation with $1 \mu M$ random 2-FAM and Apt1"-FAM. Scale bars: $25 \mu \mathrm{m}$.

\section{Characterization of the P1 dots@PCN-224 probe}

The transmission electron microscopy (TEM) images of P1 dots and metal-organic framework (MOF) nanoparticles (PCN-224) showed the spherical and monodisperse feature with an average diameter of around $11 \mathrm{~nm}$ (Fig. 4A) and $83 \mathrm{~nm}$ (Fig. 4B), respectively. The ECL probe was prepared with PCN-224 as the carrier to assemble the cDNA functionalized P1 dots through electrostatic adsorption (Scheme 1A), which could further enhance the ECL emission. Morphologic characteristics also demonstrated the successful assembly of the probe, which showed good distribution and dense loading of P1 dots on PCN224 (Fig. 4C). Moreover, the P1 dots@PCN-224 probe could maintain a stable hydrodynamic diameter (Fig. S9†), which proved the good stability of the probe. In addition, powder X-ray diffraction (PXRD) data demonstrated the pure phase of PCN224 (Fig. 4D), and positively charged PCN-224 (ref. 38) could easily adsorb negatively charged P1 dots-DNA (Fig. 4E). After loading P1 dots-cDNA, the MOFs exhibited two new peaks at 247 and $358 \mathrm{~nm}$ (Fig. 4F), attributed to the merging of MOF absorption around $224 \mathrm{~nm}$ with cDNA absorption at $255 \mathrm{~nm}$, and the absorption of $\mathbf{P 1}$ dots at $348 \mathrm{~nm}$ (Fig. 1B), respectively.

\section{Protein profiling and cell identification}

The assembly process of DNA sequences on Au/ITO for ECL imaging was examined with electrochemical impedance spectroscopy (Fig. S10†). Upon the self-assembly of HP3 and then blocking with 6-mercapto-1-hexanol $(\mathrm{MCH})$ on $\mathrm{Au} /$ indium tin oxide (ITO), the electron-transfer resistance $\left(R_{\mathrm{ct}}\right)$ successively increased. After incubating with S-DNA, the $R_{\mathrm{ct}}$ further increased, indicating the successful capture of the released $S$ DNA in the target recycling mixture.

In order to achieve highly sensitive imaging, the Apt1 concentration, digestion time, amount of Exo III, HP3 concentration and incubation time of the probe were optimized, sequentially (Fig. S11 $\dagger$ ). Under the optimized detection conditions, the probe was introduced into the DNA array for ECL imaging measurements through the hybridization of cDNA with open HP3. With increasing concentration of MUC1 and HER2, the ECL brightness increased correspondingly and depended
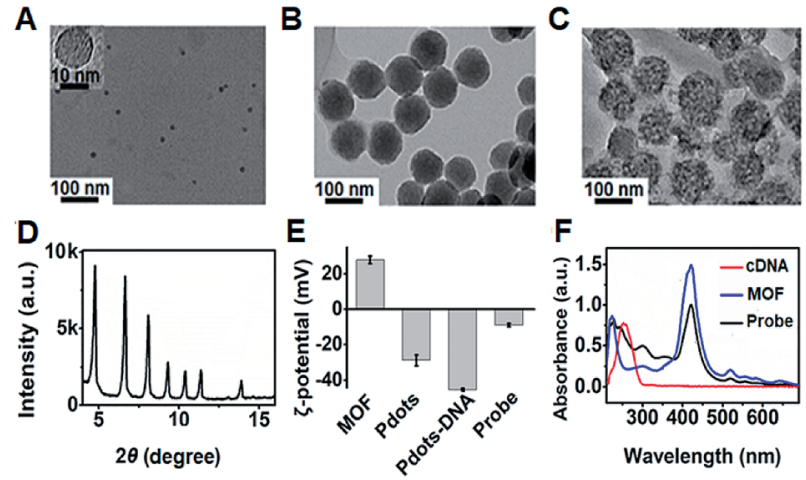

Fig. 4 TEM images of (A) P1 dots, (B) PCN-224 NPs, and (C) probe. (D) PXRD pattern of MOF nanoparticles. (E) $\zeta$-Potential characterization. (F) UV-vis absorbance of MOF NPs, cDNA, and the probe. 

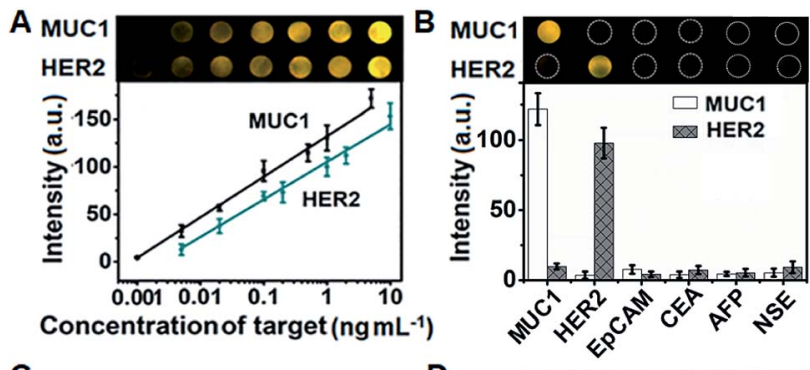

C
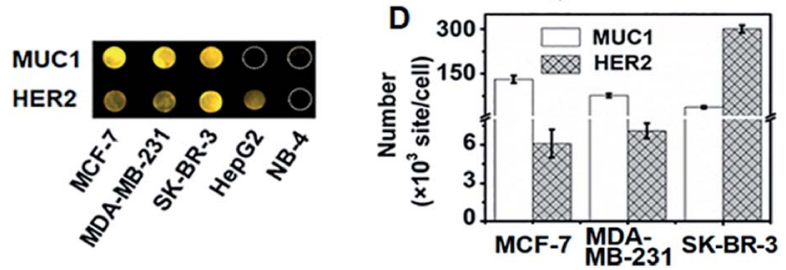

Fig. 5 (A) ECL image and calibration curves for the detection of MUC1 and HER2. (B) Specificity of the proposed strategy for imaging detection of MUC1, HER2, EpCAM, CEA, AFP and NSE at $1 \mathrm{ng} \mathrm{mL}^{-1}$. (C) $E C L$ image and (D) detection results of MUC1 and HER2 on different cells $(n=3)$.

linearly on the logarithm of the protein concentration in the range of $1 \mathrm{pg} \mathrm{mL} \mathrm{m}^{-1}$ to $5 \mathrm{ng} \mathrm{mL} \mathrm{m}^{-1}$ for MUC1 and $5 \mathrm{pg} \mathrm{mL}^{-1}$ to 10 $\mathrm{ng} \mathrm{mL} \mathrm{m}^{-1}$ for HER2 with limits of detection (LODs) of $1 \mathrm{pg} \mathrm{mL}$ and $5 \mathrm{pg} \mathrm{mL} \mathrm{m}^{-1}$ at a signal-to-noise ratio of 3 , respectively (Fig. 5A). The sensitive strategy based on triple amplification processes showed more excellent performance compared with several previous reports (Table $\mathrm{S} 4 \dagger$ ). The ECL responses to different proteins such as the epithelial cell adhesion molecule (EpCAM), carcinoembryonic antigen (CEA), alpha fetoprotein (AFP), neuron-specific enolase (NSE), MUC1 and HER2 demonstrated that the proposed imaging method exhibited high specificity to the target proteins (Fig. 5B).

The proposed strategy could be effectively extended to the quantification of membrane proteins on various types of cells. It is well known that HepG2 cells do not express MUC1, and NB-4 cells do not also overexpress MUC1 and HER2. Thus the ECL image showed obviously different responses to three kinds of breast cancer cells (MCF-7, MDA-MB-231 and SK-BR-3) (Fig. 5C). The average amounts of MUC1 on each MCF-7, MDA-MB-231 and SK-BR-3 cell were determined to be 13.1, 7.64 and $3.71 \times$ $10^{6}$ sites, while the average amounts of HER 2 on these cells were $6.1,7.1$ and $301 \times 10^{5}$, respectively (Fig. 5D). These results were close to the data reported previously. ${ }^{39,40}$ The relative standard deviation for three parallel measurements was less than $12.2 \%$ (Fig. S12 $\dagger$ ), indicating the high specificity and good accuracy of the imaging strategy.

\section{Conclusions}

A dual intramolecular RET mechanism is proposed with newly designed triple-component P1 dots to greatly enhance the ECL emission of Pdots. The P1 dots contain two pairs of energy donors and energy acceptors, which reduce the path of energy transmission and energy loss, and thus show a high ECL efficiency at a relatively low ECL potential. Through loading DNA functionalized P1 dots on MOFs as a carrier, a sensitive ECL probe has been structured for highly sensitive bioanalysis by the introduction of target-mediated enzymatic circulation amplification as the third amplification process. The proposed ECL imaging platform shows excellent performance for sensitive and specific quantification of cell surface MUC1 and HER2 with an LOD in the pg $\mathrm{mL}^{-1}$ level, and can be used for screening of breast cancer cells. By using different aptamers to specifically code the targets, the designed method can be conveniently extended for simultaneous visual analysis of different targets. This dual intramolecular RET strategy provides a new concept for ECL enhancement, and broadens the application of Pdots in ECL imaging and bioanalysis.

\section{Conflicts of interest}

The authors declare no competing financial interests.

\section{Acknowledgements}

This work was financially supported by the National Natural Science Foundation of China (21635005, 21827812, 21890741).

\section{References}

1 D. Cui, J. G. Huang, X. Zhen, J. C. Li, Y. Y. Jiang and K. Y. Pu, Angew. Chem., Int. Ed., 2019, 58, 1-6.

2 Z. H. Liu, Y. K. Yang, Z. Z. Sun and C. F. Wu, Opt. Mater., 2016, 62, 1-6.

3 K. Y. Pu, A. J. Shuhendler, J. V. Jokerst, J. G. Mei, S. S. Gambhir, Z. N. Bao and J. H. Rao, Nat. Nanotechnol., 2014, 9, 233-239.

4 B. Y. Zhang, F. Wang, H. Zhou, D. Y. Gao, Z. Yuan, C. F. Wu and X. J. Zhang, Angew. Chem., Int. Ed., 2019, 58, 2744-2748.

5 C. F. Wu and D. T. Chiu, Angew. Chem., Int. Ed., 2013, 52, 3086-3109.

6 X. Wu, Q. DeGottardi, I. C. Wu, J. B. Yu, L. Wu, F. M. Ye, C. T. Kuo, W. W. Kwok and D. T. Chiu, Angew. Chem., Int. Ed., 2017, 56, 14908-14912.

7 Y. Chen, S. W. Zhou, L. L. Li and J. J. Zhu, Nano Today, 2017, 12, 98-115.

8 R. P. Dai, F. M. Wu, H. F. Xu and Y. W. Chi, ACS Appl. Mater. Interfaces, 2015, 7, 15160-15167.

9 N. N. Wang, Y. Q. Feng, Y. W. Wang, H. X. Ju and F. Yan, Anal. Chem., 2018, 90, 7708-7714.

10 F. Sun, Z. Y. Wang, Y. Q. Feng, Y. X. Cheng, H. X. Ju and Y. W. Quan, Biosens. Bioelectron., 2018, 100, 28-34.

11 Q. Y. Lu, J. J. Zhang, Y. Y. Wu and S. H. Chen, RSC Adv., 2015, 5, 63650-63654.

12 Y. Q. Feng, N. N. Wang and H. X. Ju, Anal. Chem., 2018, 90, 1202-1208.

13 K. M. Omer, S. Y. Ku, J. Z. Cheng, S. H. Chou, K. T. Wong and A. J. Bard, J. Am. Chem. Soc., 2011, 133, 5492-5499.

14 R. Ishimatsu, S. Matsunami, T. Kasahara, J. Mizuno, T. Edura, C. Adachi, K. Nakano and T. Imato, Angew. Chem., Int. Ed., 2014, 53, 6993-6996. 
15 T. B. Gao, J. J. Zhang, R. Q. Yan, D. K. Cao, D. C. Jiang and D. J. Ye, Inorg. Chem., 2018, 57, 4310-4316.

16 X. Wei, M. J. Zhu, Z. Cheng, M. Lee, H. Yan, C. S. Lu and J. J. Xu, Angew. Chem., Int. Ed., 2019, 58, 3162-3166.

17 S. Carrara, A. Aliprandi, C. F. Hogan and L. De Cola, J. Am. Chem. Soc., 2017, 139, 14605-14610.

18 Z. Y. Wang, Y. Q. Feng, N. N. Wang, Y. X. Cheng, Y. W. Quan and H. X. Ju, J. Phys. Chem. Lett., 2018, 9, 5296-5302.

19 X. Liu, H. Jiang, J. P. Lei and H. X. Ju, Anal. Chem., 2007, 79, 8055-8060.

20 Y. M. Lei, W. X. Huang, M. Zhao, Y. Q. Chai, R. Yuan and Y. Zhuo, Anal. Chem., 2015, 87, 7787-7794.

21 S. J. Li, Y. Liu and Q. Ma, TrAC, Trends Anal. Chem., 2019, 110, 277-292.

22 Y. P. Dong, T. T. Gao, Y. Zhou and J. J. Zhu, Anal. Chem., 2014, 86, 11373-11379.

23 P. Zhang, Z. Y. Li, H. J. Wang, Y. Zhuo, R. Yuan and Y. Q. Chai, Nanoscale, 2017, 9, 2310-2316.

24 Y. Q. Feng, F. Sun, N. N. Wang, J. P. Lei and H. X. Ju, Anal. Chem., 2017, 89, 7659-7666.

25 J. L. Liu, M. Zhao, Y. Zhuo, Y. Q. Chai and R. Yuan, Chem.Eur. J., 2017, 23, 1853-1859.

26 L. Zhang, S. H. Zeng, L. X. Yin, C. Y. Ji, K. C. Li, Y. Q. Li and Y. Wang, New J. Chem., 2013, 37, 632-639.

27 S. W. Zhang, Y. Sheng, G. Wei, Y. W. Quan, Y. X. Cheng and C. J. Zhu, Polym. Chem., 2015, 6, 2416-2422.
28 C. H. Dai, D. L. Yang, X. Fu, Q. M. Chen, C. J. Zhu, Y. X. Cheng and L. H. Wang, Polym. Chem., 2015, 6, 50705076.

29 R. Yoshii, A. Hirose, K. Tanaka and Y. Chujo, J. Am. Chem. Soc., 2014, 136, 18131-18139.

30 Z. X. Cai, F. M. Li, W. Xu, S. J. Xia, J. B. Zeng, S. G. He and X. Chen, Nano Res., 2018, 11, 1447-1455.

31 X. Tan, B. Zhang and G. Z. Zou, J. Am. Chem. Soc., 2017, 139, 8772-8776.

32 Z. G. Han, et al., Angew. Chem., Int. Ed., 2019, 58, 5915-5919.

33 Y. P. He, L. Q. Yang, F. Zhang, B. Zhang and G. Z. Zou, J. Phys. Chem. Lett., 2018, 9, 6089-6095.

34 N. Myung, Z. F. Ding and A. J. Bard, Nano Lett., 2002, 2, 13151319.

35 A. Schreivogel, J. Maurer, R. Winter, A. Baro and S. Laschat, Eur. J. Org. Chem., 2006, 2006, 3395-3404.

36 Y. Wang, R. Du, L. Qiao and B. Liu, Chem. Commun., 2018, 54, 9659-9662.

37 K. S. Kim, J. Kim, D. H. Kim, H. S. Hwang and K. Na, Biomater. Sci., 2018, 6, 1217-1226.

38 J. Park, Q. Jiang, D. W. Feng, L. Q. Mao and H. C. Zhou, J. Am. Chem. Soc., 2016, 138, 3518-3525.

39 S. Q. Li, Y. R Liu, L. Liu, Y. M. Feng, L. Ding and H. X. Ju, Angew. Chem., Int. Ed., 2018, 57, 12007-12011.

40 T. Gao, B. Wang, L. Shi, X. L. Zhu, Y. Xiang, J. I. Anzai and G. X. Li, Anal. Chem., 2017, 89, 10776-10782. 